CALT-68-2818

\title{
Phase transitions in symmetric orbifold CFTs and universality
}

\author{
Christoph A. Keller* \\ California Institute of Technology, \\ Pasadena, CA 91125, USA
}

January 2011

\begin{abstract}
Since many thermodynamic properties of black holes are universal, the thermodynamics of their holographic duals should be universal too. We show how this universality is exhibited in the example of symmetric orbifolds of general two dimensional CFTs. We discuss the free energies and phase diagrams of such theories and show that they are indeed universal in the large $N$ limit. We also comment on the implications of our results for the classification of CFTs that can have an interpretation as holographic duals to gravity theories on $A d S_{3}$.
\end{abstract}

${ }^{*}$ E-mail: ckeller@theory.caltech.edu 


\section{Introduction and overview}

\section{$1.1 \quad$ Introduction}

Investigating the thermodynamic properties of black holes has a long history. A common theme in these investigations is that many black holes are characterized by very few parameters, and that their thermodynamic properties are universal. Another theme is the search for an underlying microscopic description of the thermodynamics. A very fruitful approach to this has been the application of the AdS/CFT correspondence [1]. In view of this, it should be possible to see this universality also from the holographic dual theory of the black hole.

In this note we will concentrate on one specific class of such holographic dual CFTs, namely symmetric orbifolds of two dimensional CFTs. In many string configurations leading to $A d S_{3}$ geometries, such as D1-D5 systems, the holographic dual conformal field theories are given by (deformations of) symmetric orbifold CFTs [2]. In this particular case, the D1 branes are considered as instantons of the low energy theory on the D5 branes, whose moduli space is then a deformation of the symmetric product of the moduli space of a single instanton [3, 4]. It is thus very natural to investigate thermodynamic properties of symmetric orbifold CFTs.

The fact that we are dealing with CFTs in two dimensions will be very helpful for our analysis, as it allows us to use methods specific to $d=2$. One crucial property that will be central to our analysis is modular invariance of the partition function. This allows us to relate the free energy for a different ranges of temperature and other parameters to each other. In particular it is then sufficient to know the behavior of the theory at low temperature to also obtain the high temperature behavior.

In the remainder of this section, we will make some general remarks about the free energy and phase transitions of $2 \mathrm{~d}$ CFTs. In section 2 we use the methods described above to analyze properties of the free energy of symmetric orbifold CFTs. We will discuss the phase diagram of their Hawking-Page transitions [5], the behavior near the phase transitions, and the critical exponents for a class of order parameters. In particular we will show that many of those properties are indeed universal, and do not depend on the underlying theory.

Even though our analysis is for general, not necessarily holomorphic theories, we will also briefly discuss the behavior of the elliptic genus for $N=2$ theories. In section 3 we will also discuss what criteria general theories that are not symmetric orbifolds have to satisfy if they are to exhibit the same universal behavior.

\subsection{Some general remarks on two dimensional CFTs}

Consider a conformal field theory in two dimensions with left- and right-moving central charges such that $c_{L}-c_{R}=0 \bmod 24$. We will assume that the CFT has a discrete spectrum with finite multiplicities, and that its vacuum is unique. Also we will only consider unitary theories, so that all states have positive norm and non-negative weight. We also define the theory on a circle whose radius sets the unit of length. By the usual arguments of QFT at finite temperature, we can then obtain the partition function as the vacuum amplitude on the torus, whose modulus $\tau$ is fixed by the temperature and the spin potential. 
More precisely, we define the partition function as

$$
Z(\tau, \bar{\tau})=\operatorname{Tr} q^{L_{0}-c_{L} / 24} \bar{q}^{\bar{L}_{0}-c_{R} / 24}=\sum_{m, \bar{m} \in I} d(m, \bar{m}) q^{m} \bar{q}^{\bar{m}} .
$$

As usual we have defined

$$
q=e^{2 \pi i \tau}=e^{-\beta+i \mu} .
$$

Here $\beta$ is the inverse temperature and the spin potential $\mu$ is the variable conjugate to the momentum on the circle. We will often set $\mu=0$ and focus on the behavior of $Z$ as a function of $\beta$.

For reasons that will become clear in a moment, we have defined the vacuum to have energy $-c / 24$. When discussing physical quantities, it will often be useful to shift the vacuum energy to zero. We will denote quantities which are shifted in such a way by a tilde. In particular we define

$$
\tilde{Z}(\tau, \bar{\tau})=q^{c_{L} / 24} \bar{q}^{c_{R} / 24} Z(\tau, \bar{\tau})=\sum_{m, \bar{m} \in \tilde{I}} \tilde{d}(m, \bar{m}) q^{m} \bar{q}^{\bar{m}}
$$

where $\tilde{d}(m, \bar{m})$ is the number of states at left- and right-moving levels $(m, \bar{m})$. Finally, let us define the free energy $F$ as

$$
Z(\tau, \bar{\tau})=e^{-2 \pi \operatorname{Im}(\tau) F(\tau, \bar{\tau})}=e^{-\beta F(\tau, \bar{\tau})} .
$$

Similarly, define $\tilde{F}$ as the logarithm of $\tilde{Z}$.

Many of the properties of $Z$ follow from general principles. As mentioned above, the partition function $Z$ corresponds to the vacuum amplitude of the Euclidean theory on the torus. As the theory is conformal, it only depends on the conformal structure. The partition function $Z$ must therefore be invariant under the modular group $\Gamma=S L(2, \mathbb{Z})$. Note that this holds only for $Z$, and not for the shifted $\tilde{Z}$. It follows that we only need to know $F$ in one fundamental region of $\Gamma$. For all other values of $\tau$ we can simply apply the appropriate modular transformation. For example for $\mu=0$ we can use the modular transformation $S$ to get

$$
Z(\beta)=Z\left(4 \pi^{2} / \beta\right)
$$

The behavior of $F$ for very low temperatures is dominated by the vacuum contribution, and by using (1.5) we can also obtain the behavior at very high temperature,

$$
F(\beta) \simeq\left\{\begin{array}{cl}
-\frac{\left(c_{L}+c_{R}\right)}{24} & \text { for } \beta \gg 1, \\
-\frac{c_{L}+c_{R}}{24} \frac{4 \pi^{2}}{\beta^{2}} & \text { for } \beta \ll 1 .
\end{array}\right.
$$

The behavior in the high and low temperature limit is thus universal and depends only on the central charges. The behavior between this limits on the other hand is of course not universal and depends on the spectrum of the CFT. The only thing we know is that it is smooth and monotonically decreasing. One of the main results of our analysis will be that for symmetric orbifolds the temperature region where the behavior is universal extends towards the self-dual temperature $\beta=2 \pi$ as $N$ grows large. In the limit of $N \rightarrow \infty$, the free energy becomes universal for all temperatures. 
In fact, the modular transformation we have discussed can be used to obtain the asymptotic growth of the number of states. For $m$ and $\bar{m}$ very large it is given by Cardy's formula,

$$
d(m, \bar{m}) \sim \exp \left(2 \pi \sqrt{\frac{c_{L} m}{6}}\right) \exp \left(2 \pi \sqrt{\frac{c_{R} \bar{m}}{6}}\right),
$$

where we have only given the leading exponential growth. It follows from this formula that the infinite sum in (1.1) converges absolutely, so that $\log Z$ and all its derivatives are continuous functions of temperature.

\subsection{Hagedorn transitions and Hawking-Page transitions}

That $\log Z$ and all its derivatives are continuous functions of $\beta$ is to be expected on even more general grounds. A quantum field theory with finitely many local degrees of freedom cannot have a phase transition at finite volume. Phase transitions can, however, occur if the number of degrees of freedom is taken to infinity. In particular the free energy can diverge above a certain temperature, so that a Hagedorn transition occurs.

We will therefore look for phase transitions in families $\left\{\mathcal{C}^{(N)}\right\}_{N \in \mathbb{N}}$ of CFTs whose central charges are given by $c_{L, R}(N)=c_{L, R} N$. Plugging this into (1.5), we see immediately that for large enough temperatures the free energy is proportional to $N$, which means that for $N=\infty$ the free free energy indeed starts to diverge above some Hagedorn temperature $T_{H}$.

Let us briefly discuss how to interpret this Hagedorn transition in the context of the AdS/CFT correspondence. The natural units on $A d S_{3}$ are given in terms of the AdS radius $R$. Since $R \sim N$, we introduce the rescaled free energy

$$
f_{N}(\tau)=\frac{1}{N} F_{N}(\tau), \quad f(\tau)=\lim _{N \rightarrow \infty} f_{N}(\tau)
$$

Note that we have not shifted the vacuum energy, so that the energy of empty AdS space is $-\left(c_{L}+c_{R}\right) / 24$. As usual, $f_{N}$ is analytic, but due to the limit $f$ can exhibit phase transitions. Such a transition corresponds to a Hawking-Page transition: below the critical temperature, the free energy is dominated by the contribution of the $A d S$-vacuum. Above it, the entropy of the BTZ black holes gives the dominating contribution.

We will show that for symmetric orbifold theories the Hagedorn transition occurs at $T_{H}=\frac{1}{2 \pi}$. In the corresponding gravity theory there is then a Hawking-Page transition at that temperature. More precisely, we will show that the free energy is given by

$$
f(\beta)=\left\{\begin{array}{cl}
-\frac{c_{L}+c_{R}}{24} & : \quad \beta>2 \pi \\
-\frac{4 \pi^{2}}{\beta^{2}} \frac{c_{L}+c_{R}}{24} & : \quad \beta<2 \pi
\end{array} .\right.
$$

As a general remark note that $\tilde{F}$ is the quantity that appears naturally when analyzing phase transitions of the conformal field theory in its own right, whereas $f$ is the natural quantity for the analysis of the CFT as a holographic dual to the gravity side. Of course all important properties of $f$ can be reconstructed from $\tilde{F}$ and vice versa, but depending on the context one or the other will be more appropriate when doing computation. 


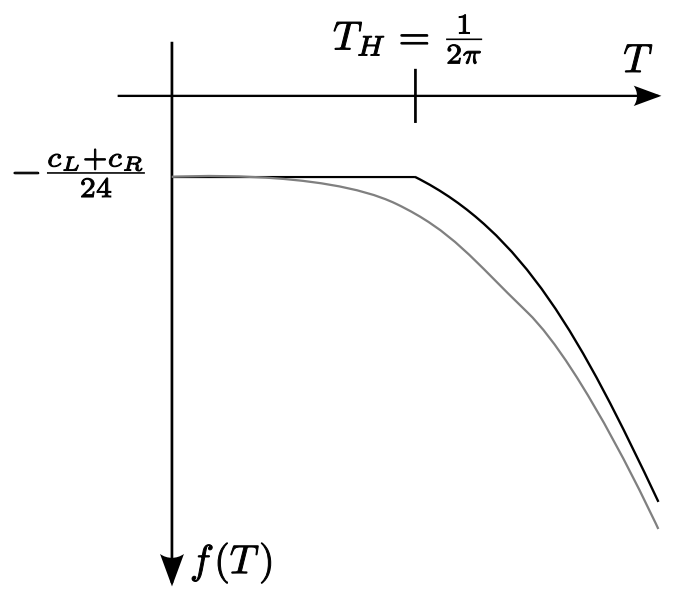

Figure 1: A generic CFT (gray line) has no phase transition and is universal only for very high and very low temperatures. The symmetric orbifold for $N=\infty$ (black line) is universal at all temperatures and has a first order phase transition at $T_{H}=\frac{1}{2 \pi}$.

\subsection{The phase diagram}

Let us briefly sketch why one expects exactly such a Hawking-Page phase transition for large $N$ theories. As a toy model we take a holomorphic theory with $c_{L}=24 \mathrm{~N}$ and consider only the contribution $q^{-N}$ of the vacuum, neglecting all higher weight states. This is clearly not a modular invariant partition function, so to render it modular invariant, we sum over all images of $\Gamma=S L(2, \mathbb{Z}) / B(\mathbb{Z})$, where $B(\mathbb{Z})$ is the translation subgroup generated by $T$. We obtain *

$$
Z(\tau)=\sum_{(c, d)=1} e^{-2 \pi i N \frac{a \tau+b}{c \tau+d}} .
$$

If we tessellate the upper half plane into fundamental regions of $\Gamma$ as in figure 2, then for every fundamental region there is an element $\left(\begin{array}{ll}a & b \\ c & d\end{array}\right) \in \Gamma$ so that the corresponding term in the sum is dominant. When we move from one fundamental region to another, a different term becomes dominant. In particular when we send $N \rightarrow \infty$, a phase transition will occur on the boundary between the regions. One might thus expect the phase diagram to look like the left diagram in figure 2. On the other hand it is straightforward to see that $F$ is invariant under $\tau \mapsto \tau+1$, so that the phase space diagram should be given by the right diagram in figure 2 [6].

The Poincaré sum (1.10) has a very natural interpretation on the gravity side. The classical solutions of Euclidean gravity with fixed conformal boundary $\tau$ are essentially given by BTZ black holes which correspond to quotients of the AdS vacuum by elements of $S L(2, \mathbb{Z})$ [7. When evaluating the Euclidean gravity path integral, we are instructed to sum over all such classical solutions, which gives the Poincaré sum (1.10). (See [6, 8] for a more detailed discussion.) The structure of the phase diagram can thus be explained by the different BTZ black holes which dominate the free energy in different regions of phase space.

*This sum is divergent and needs to be regularized. We will ignore this issue since it does not affect the point we are making. 


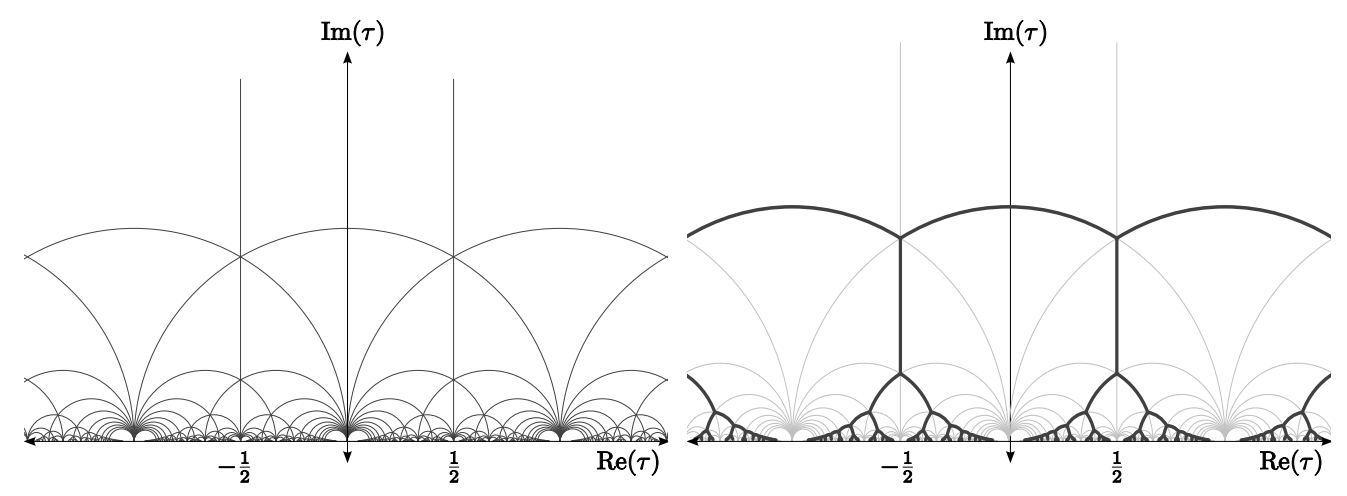

Figure 2: To the left, the tessellation of the upper half plane into fundamental regions of $S L(2, \mathbb{Z})$. To the right, the proposed phase diagram, taking into account the invariance under $T$. (Figures taken from [9].)

For instance, if we restrict to $\mu=0$ we can restrict the sum over images to

$$
Z_{N}=e^{N \beta}+e^{N 4 \pi^{2} / \beta}
$$

The free energy then has exactly the behavior (1.9).

There are two problems with this argument. First, it only works for holomorphic partition functions. We have made use of the mathematical fact that a holomorphic modular form can be reconstructed by taking the Poincaré sum (1.10) of its polar part. No analogue theorem exists for non-holomorphic modular forms.

Second, a CFT necessarily contains many more states than just the vacuum. In a family of CFTs it is thus possible that the contribution of these states smoothes out the phase transition coming from the vacuum term. A simple example that shows that this can happen is the family of $N$ tensor products of a CFT. In this case the partition function is simply the $N$ th power of the original partition function, and

$$
f(\beta)=f_{N}(\beta)=f_{1}(\beta)
$$

which is analytic in $\beta$.

In fact, if we want the full theory to exhibit the same behavior as (1.10), it is straightforward to see that the number of states $\tilde{d}_{N}(m, \bar{m})$ in the family has to satisfy the criterion

$$
\lim _{N \rightarrow \infty} \frac{1}{N} \log \left(1+\sum_{m, \bar{m}} \tilde{d}_{N}(m, \bar{m}) e^{-2 \pi(m+\bar{m})}\right)=0 .
$$

This follows from the requirement that the contributions of the higher level terms must be subleading in $N$. This is where the symmetric orbifold becomes important. Starting from the tensor theory described above, it projects out most of the low lying states, such that the resulting theory does indeed exhibit phase transitions. 


\section{Universal properties of large $N$ symmetric orbifolds}

\subsection{Symmetric orbifolds}

We start out with the partition function of an arbitrary seed theory $\mathcal{C}_{1}$ (which satisfies the technical conditions outlined in the introduction),

$$
Z\left(\mathcal{C}_{1}\right)=\sum_{m, \bar{m} \in I} d(m, \bar{m}) q^{m} \bar{q}^{\bar{m}}
$$

We assume that there is indeed a discrete set of states $I$, and that lowest lying state, the vacuum, occurs exactly once.

As we have seen above, for the family of $N$ fold tensor products of the seed theory $\mathcal{C}_{1}, f$ does not exhibit any phase transitions, since the higher lying states smoothen out the free energy. To eliminate as many of those states as possible, we need to perform an orbifold. As the tensor product is symmetric under permutations of its factors, we can orbifold with respect to the permutation group $S_{N}$. This orbifold projects out most of the low lying states of the theory. As in any orbifold however, it also introduces new twisted sectors that are given by the conjugacy classes of the orbifold group. For $S_{N}$ these are simply the product of cycles

$$
(1)^{N_{1}}(2)^{N_{2}} \cdots(s)^{N_{s}}, \quad \sum_{n} n N_{n}=N
$$

In those twisted sectors we again need to project out states which are not invariant under the stabilizer subgroup of the conjugacy classes, which in this case is given by the semidirect product of cyclic permutations $\mathbb{Z}_{n}$ within a given cycle and permutations of different cycles of the same length. As is often the case in such computations, it turns to be easier to compute the grand canonical ensemble, i.e. the generating function of the partition functions [10, 11, 12 .

$$
\mathcal{Z}=\sum_{N \geq 0} p^{N} Z\left(S^{N} \mathcal{C}_{1}\right)=\prod_{n>0} \prod_{m, \bar{m} \in I}\left(1-p^{n} q^{m / n} \bar{q}^{\bar{m} / n}\right)^{-d(m, \bar{m}) \delta_{m-\bar{m}}^{(n)}}
$$

Alternatively, to make the modular properties of the partition functions manifest, we can also express $\mathcal{Z}$ in terms of Hecke operators,

$$
\mathcal{Z}=\exp \left(\sum_{L>0} \frac{p^{L}}{L} T_{L}^{\prime} Z(q, \bar{q})\right)
$$

where the action of the Hecke operator $T_{L}^{\prime}$ is defined as

$$
T_{L}^{\prime} f(\tau, \bar{\tau})=\sum_{d \mid L} \sum_{b=0}^{d-1} f\left(\frac{L \tau+b d}{d^{2}}, \frac{L \bar{\tau}+b d}{d^{2}}\right)
$$

We have put a prime on $T_{L}$ as a reminder that this definition differs in normalization from the standard Hecke operator. 


\subsection{Universal properties}

We will now show that in the limit $N \rightarrow \infty$, these families of theories have universal properties. Universal means that they only depend on the central charge of the seed theory $\mathcal{C}_{1}$, but not on any other properties of its spectrum. We will find the following universal properties:

1. The number of states always grows as $\tilde{d}_{\infty}(m, \bar{m}) \sim e^{2 \pi m} e^{2 \pi \bar{m}}$.

2. The divergent part of the free energy near the Hagedorn transition is universal. In particular, as we will show later on, this implies that the critical exponent for the phase transitions of certain families of order parameters is always 1 .

3. The phase diagram for the rescaled free energy $f(\tau)$ is universal, and is given by figure 2

Let us now show these properties. For our analysis it will be easiest to work with the shifted free energy $\tilde{F}$, and then translate our results into statements about $f$ if needed.

\section{Growth of states}

Let us find an expression for the growth of the number of states of the symmetric orbifold theory of large $N$. First, we show that the partition function $\tilde{Z}_{\infty}$ of the $N=\infty$ theory exists, i.e. the number of states $\tilde{d}_{\infty}(m, \bar{m})=\lim _{N \rightarrow \infty} \tilde{d}_{N}(m, \bar{m})$ remains finite. More precisely, we will show that

$$
\tilde{d}_{\infty}(m, \bar{m}) \sim e^{2 \pi m} e^{2 \pi \bar{m}} \quad \text { for } m \gg c_{L}, \bar{m} \gg c_{R} .
$$

This behavior is certainly consistent with a Hagedorn transition at $\beta=2 \pi$. In fact, (2.6) is also true for finite $N$, as long as $m, \bar{m}$ are sufficiently smaller than $N$.

To show (2.6), let us write the action of the Hecke operators on $Z$ as

$$
T_{L}^{\prime} Z(q, \bar{q})=q^{-c_{L} L / 24} \bar{q}^{-c_{R} L / 24}\left(1+\sum_{m, \bar{m}>0} \tilde{d}_{T_{L}}(m, \bar{m}) q^{m} \bar{q}^{\bar{m}}\right) .
$$

From the definition of the Hecke operator it follows that the sum in (2.7) runs over $m, \bar{m} \geq$ $m_{0} L$ for some positive $m_{0}$.

Introducing $\tilde{p}=p q^{-\frac{c_{L}}{24}} \bar{q}^{-\frac{c_{R}}{24}}$, we can write (2.4) as

$$
\begin{aligned}
\exp \left(\sum_{L>0} \frac{1}{L} \tilde{p}^{L}+\sum_{L>0} \frac{1}{L} \tilde{p}^{L} \sum_{m, \bar{m} \geq m_{0} L} \tilde{d}_{T_{L}}(m, \bar{m}) q^{m} \bar{q}^{\bar{m}}\right) \\
=\left(\sum_{K \geq 0} \tilde{p}^{K}\right) \exp \left(\sum_{L>0} \frac{1}{L} \tilde{p}^{L} \sum_{m, \bar{m} \geq m_{0} L} \tilde{d}_{T_{L}}(m, \bar{m}) q^{m} \bar{q}^{\bar{m}}\right) .
\end{aligned}
$$

The sought-after number of states $\tilde{d}_{N}(m, \bar{m})$ is given by the coefficient of the term $\tilde{p}^{N} q^{m} \bar{q}^{\bar{m}}$. Since the exponential on the right contributes only non-negative powers of $\tilde{p}$, we can restrict 
the sum on the left to $0 \leq K \leq N$. Pulling out a factor of $\tilde{p}^{N}$, we need to find the coefficient of $\tilde{p}^{0} q^{m} \bar{q}^{\bar{m}}$ of

$$
\sum_{K=0}^{N} \tilde{p}^{-K} \exp \left(\sum_{L>0} \frac{1}{L} \tilde{p}^{L} \sum_{m, \bar{m} \geq m_{0} L} \tilde{d}_{L}(m, \bar{m}) q^{m} \bar{q}^{\bar{m}}\right) .
$$

Let us analyze the contribution for a given value of $K$. We consider a partition $K=\sum_{j} L_{j}$, and then pick values $m_{j}, \bar{m}_{j}$ such that $m=\sum_{j} m_{j}, \bar{m}=\sum_{j} \bar{m}_{j}$. Note that this gives an upper bound for $K$,

$$
K=\sum_{j} L_{j} \leq \frac{1}{m_{0}} \sum_{j} m_{j}=\frac{m}{m_{0}}
$$

and similarly for $\bar{m}$. It follows that for $m$ or $\bar{m}$ smaller than $m_{0} N, \tilde{d}_{N}(m, \bar{m})$ is independent of $N$. In particular, this shows that the $N \rightarrow \infty$ is well-defined, and can in fact be obtained from the coefficient of a finite orbifold with $N$ large enough.

To obtain (2.6), we need to analyze (2.9) in more detail. For a start, let us consider only the linear expansion of the exponential in (2.9), which gives a total contribution of

$$
\sum_{L=1}^{N} \frac{1}{L} \tilde{d}_{L}(m, \bar{m})
$$

To estimate the contribution of this sum, we can use the fact that the $T_{L}^{\prime} Z$ is again a modular form. This means that we can approximate $\tilde{d}_{L}$ by the Cardy formula to get the asymptotic behavior

$$
\tilde{d}_{L}(m, \bar{m}) \sim \exp \left(2 \pi \sqrt{\frac{c_{L} L}{6}\left(m-\frac{c_{L} L}{24}\right)}\right) \exp \left(2 \pi \sqrt{\frac{c_{R} L}{6}\left(\bar{m}-\frac{c_{R} L}{24}\right)}\right) .
$$

(We will discuss the validity of this approximation shortly.) We see that this expression is maximized for $L=\frac{12 m}{c_{L}}$, where it contributes

$$
\tilde{d}(m, \bar{m}) \sim e^{2 \pi m} e^{2 \pi \bar{m}} .
$$

We should now estimate the contributions of all other terms as well and check if their total contribution makes (2.13) grow even faster. The results of the next section show however that there is no Hagedorn transition for $\beta$ bigger then $2 \pi$. This shows that the leading behavior of (2.13) does not change when taking into account the other contributions.

Note that we have used the Cardy formula for $m=\frac{c}{12}$, which is not in its usual range of validity, which requires $m \gg c$. Note however that by the arguments given above, $T_{L}^{\prime} Z$ has a gap which is parametrically large in $L$. One can show that in this case the Cardy formula already holds for $m>\frac{c}{24}$ (see appendix A.1).

\section{The free energy $\tilde{F}(\beta)$}

Let us now discuss in which sense the free energy $\tilde{F}_{N}$ is universal in the large $N$ limit. In a first step, consider the case $\mu=0$. We need to determine the coefficient of $\tilde{p}^{0}$ of (2.9). We can evaluate the sum over $m$ by using a saddle point approximation,

$$
\int d m\left(\frac{c_{L} L}{96\left(m-\frac{c_{L} L}{24}\right)^{3}}\right)^{1 / 4} e^{2 \pi \sqrt{\frac{c_{L} L\left(m-\frac{c_{L}}{6}\right)}{6}}} e^{-\beta m}=1 \cdot e^{\left(\frac{4 \pi^{2}}{\beta}-\beta\right) \frac{c_{L} L}{24}}+\ldots .
$$


Here we have used the Cardy formula to express $\tilde{d}(m, \bar{m})$, which is permissible for large $L$ since the saddle point is at $m_{0}=\left(4 \pi^{2} \beta^{-2}+1\right) c L / 24+O(1)$. The total subleading corrections to all these manipulations are of order $O\left(e^{-m_{0} L \beta}\right)$; this includes the contribution of terms with small $L$, where the use of the Cardy formula is not permitted.

Including the right movers but neglecting all subleading terms for the moment (2.9) gives

$$
\sum_{K=0}^{N} \tilde{p}^{-K} \exp \left(-\log \left(1-\tilde{p} e^{\left(\frac{4 \pi^{2}}{\beta}-\beta\right)\left(c_{L}+c_{R}\right) / 24}\right)\right),
$$

so that we can read off from the $\tilde{p}^{0}$ coefficient

$$
\tilde{Z}_{N}(\beta)=\sum_{L=0}^{N} e^{L\left(\frac{4 \pi^{2}}{\beta}-\beta\right)\left(c_{L}+c_{R}\right) / 24}=\frac{e^{(N+1)\left(\frac{4 \pi^{2}}{\beta}-\beta\right)\left(c_{L}+c_{R}\right) / 24}-1}{e^{\left(\frac{4 \pi^{2}}{\beta}-\beta\right)\left(c_{L}+c_{R}\right) / 24}-1} .
$$

To include the subleading corrections, we use the fact that they are $O\left(e^{-n_{0} L \beta}\right)$, so that the result is changed only by multiplication with a regular function $e^{-\beta \tilde{F}_{N}^{r e g}(\beta)}$. In total we thus obtain

$$
\tilde{Z}_{N}(\beta)=\frac{e^{(N+1)\left(\frac{4 \pi^{2}}{\beta}-\beta\right)\left(c_{L}+c_{R}\right) / 24}-1}{e^{\left(\frac{4 \pi^{2}}{\beta}-\beta\right)\left(c_{L}+c_{R}\right) / 24}-1} \cdot e^{-\beta \tilde{F}_{N}^{r e g}(\beta)} .
$$

The function $\tilde{F}_{N}^{r e g}(\beta)$ depends on $N$, but it is bounded uniformly in $\beta$ as long as $\beta$ is not too big.

From (2.17) we can read off the behavior of the free energy in the large $N$ limit. For $\beta<2 \pi$ we find that

$$
\tilde{F}_{N}(\beta)=-N \frac{c_{L}+c_{R}}{24}\left(\frac{4 \pi^{2}}{\beta^{2}}-1\right)+O(1)
$$

On the other hand, for $\beta>2 \pi$ we find that

$$
\tilde{F}_{\infty}(\beta)=\tilde{F}_{\infty}^{r e g}(\beta)+\beta^{-1} \log \left(1-\exp \left[\frac{\left(c_{L}+c_{R}\right)}{24}\left(\frac{4 \pi^{2}}{\beta}-\beta\right)\right]\right),
$$

where $\tilde{F}_{\infty}^{r e g}$ is a regular function whose specifics depend on the seed theory.

\section{The general phase diagram}

The above analysis shows that for $N$ going to infinity there is indeed a Hagedorn transition at the temperature $T=\frac{1}{2 \pi}$. It also shows that for large but finite $N$ the free energy $\tilde{F}_{N}$ diverges as $N$ above that temperature. It follows that the rescaled free energy $f=\lim _{N \rightarrow \infty} N^{-1} F_{N}$ should be well defined at all temperatures, and that it should have a first order phase transition at $T=\frac{1}{2 \pi}$. Its behavior as a function of $T$ looks exactly like figure 1 .

We now turn to the case of non-vanishing spin potential $\mu$. In particular, we want to show that the full phase diagram of $f(\tau)$ is given by figure 2. To do that, we use the fact that $Z_{N}(\tau)$ is modular invariant. In particular this means that if we know $f(\tau)$ in the fundamental region $\mathcal{F}_{0}$, we can obtain its value for any value of $\tau$ by modular transformations.

More precisely, we will show that in the $N \rightarrow \infty$ limit, $f(\tau)$ in the fundamental region $\mathcal{F}_{0}$ is given by the contribution of the vacuum only, i.e.

$$
f(\tau)=-\frac{\left(c_{L}+c_{R}\right)}{24}: \quad \tau \in \mathcal{F}_{0} .
$$


If (2.20), it then follows immediately that the phase diagram is indeed given by figure 2 , as the free energy is not smoothed out around the phase transition lines. To show (2.20), we need to show any corrections coming from higher lying states are negligible. This is equivalent to showing that $\tilde{F}_{\infty}(\tau)$ is finite in the fundamental region $\mathcal{F}_{0}$.

To do this we first need to extract the partition function $\tilde{Z}_{\infty}(\tau, \bar{\tau})$. To do this, we use a trick from [13. If we define $\tilde{p}:=p q^{-c_{L} / 24} \bar{q}^{-c_{R} / 24}$, then the $\tilde{Z}_{N}$ are the coefficients of the expansion of $\mathcal{Z}$ in terms of $\tilde{p}$. On the other hand we can rewrite $\mathcal{Z}$ as

$$
\begin{aligned}
\mathcal{Z}=(1-\tilde{p})^{-1} \prod_{n>0, m, \bar{m} \in I}^{\prime}\left(1-\tilde{p}^{n} q^{m / n+n c_{L} / 24} \bar{q}^{\bar{m} / n+n c_{R} / 24}\right)^{-d(m, \bar{m}) \delta_{m-\bar{m}}^{(n)}} & \\
= & (1-\tilde{p})^{-1} R(\tilde{p}),
\end{aligned}
$$

where the primed product omits the factor with $\left(n=1, m=-c_{L} / 24, \bar{m}=-c_{R} / 24\right)$. Note that we have assumed that the vacuum is unique, i.e. $d\left(-c_{L} / 24,-c_{R} / 24\right)=1$. If the expansion of $R$ is given by $R(\tilde{p})=\sum_{k} a_{k} \tilde{p}^{k}$, then $\tilde{Z}_{N}=\sum_{k=0}^{N} a_{k}$. It follows that

$$
\tilde{Z}_{\infty}=\sum_{k=0}^{\infty} a_{k}=R(1)
$$

so that the shifted free energy is given by $\tilde{F}_{\infty}$,

$$
-\beta \tilde{F}_{\infty}=\log \tilde{Z}_{\infty}=-\sum_{n>0} \sum_{m, \bar{m}}^{\prime} d(m, \bar{m}) \delta_{m-\bar{m}}^{(n)} \log \left(1-q^{m / n+n c_{L} / 24} \bar{q}^{\bar{m} / n+n c_{R} / 24}\right)
$$

It is shown in appendix A.2 that this expression indeed converges if $\tau$ is in the standard fundamental region $\mathcal{F}_{0}$. We can then translate this into a statement about the behavior of $f(\tau)$, from which (2.20) and the phase diagram in figure 2 immediately follow.

\section{3 $S U(N)$ gauge theories: comparison to $d>2$}

Let us briefly explain how our results fit in with the analysis of $S U(N)$ gauge theories in more than 2 dimensions. In particular we will compare our findings with the results of [14, 15. It turns out that although our methods are completely different, the results are very similar.

In our case the boundary CFT lives on $T^{2}=S^{1} \times S^{1}$, where the time direction has been (thermally) compactified. In [15] $S U(N)$ CFTs on $S^{1} \times S^{d-1}$ are considered. The rank of the gauge group then corresponds to $N$ of the orbifold, and the symmetric orbifold theory corresponds to the free $S U(N)$ gauge theory.

Let us first consider a non-conformal theory which is asymptotically free, such as pure Yang-Mills. In the free theory [15] also find two phases, separated by the Hagedorn temperature $T_{H}$. In the lower phase, which is interpreted as the confining phase, the free energy grows as $N^{0}$, since the degrees of freedom are (colorless) glueballs. This agrees perfectly with (2.19).

For higher temperatures, in the deconfined phase the gluons are the physical degrees of freedom, so that the free energy scales as $N^{2}$. This is slightly different from (2.18), where the free energy grows as $N^{1}$. We can see where this linear dependence comes from by considering e.g. a system of $Q_{1} \mathrm{D} 1$ and $Q_{5} \mathrm{D} 5$ branes, which in the $\mathrm{UV}$ is given by a $U\left(Q_{1}\right) \times U\left(Q_{5}\right)$ 
gauge theory. When flowing to the IR, the gauge groups are eliminated, leaving a symmetric orbifold with $N=Q_{1} Q_{5}$. Moreover, a careful counting analysis shows [16] that the $(1,1)$ strings and the $(5,5)$ strings are eliminated by D-term constraints and gauge fixing, so that only the massless $(1,5)$ modes survive. The number of degrees of freedom of the resulting effective CFT is thus proportional to $Q_{1} Q_{5}$.

Moreover, according to [15] in the high-temperature phase, the free energy grows as $F=N^{2} f(T)$ with $F(T) \sim T^{d}$, whereas we find $F \sim N T^{2}$. In the low-temperature phase, the density of states should grow Hagedorn-like, i.e. $d(n) \sim e^{n / T_{H}}$, which agrees with (2.13).

Note that our situation is slightly different, as we investigate a conformal theory. It is clear that on flat space it cannot have a phase transition, as there is no energy scale. The energy scale is given by the radius of the $S^{1}$ on which we compactify. In [18] there is an analogous discussion of the $d=4, \mathcal{N}=4$ SYM case, which is also conformal. Compactified on a finite volume, for purely kinematic reasons one finds the same behavior as above, which indicates that (in the large $N$ limit) there are also two phases, although the low temperature phase has nothing to do with confinement. In fact, uncompactifying the $S^{3}$ shows that $\mathcal{N}=4 \mathrm{SYM}$ is always in the unconfined phase on flat space.

\section{$2.4 \quad$ Elliptic genus}

Our analysis so far was for general bosonic theories whose partition functions were nonholomorphic. The original analysis [6] however used methods that are only applicable for holomorphic quantities. One way to obtain a holomorphic object is to take a theory with $N=2$ supersymmetry, and analyze its elliptic genus. As we will show, the elliptic genus (or rather the NS partition function obtained from it) has a phase diagram which is almost the same as we obtained in the bosonic case, the only difference being the underlying modular group.

Consider a seed theory with $N=2$ supersymmetry. Its elliptic genus is defined as

$$
\chi(q, y)=\operatorname{Tr}_{R R} q^{L_{0}-c / 24} \bar{q}^{\bar{L}_{0}-c / 24} y^{J_{0}}(-1)^{F}(-1)^{\bar{F}}=\sum_{m \geq 0, \ell} c(m, \ell) q^{m} y^{\ell} .
$$

Because of the insertion of $(-1)^{\bar{F}}$, the right movers only give a constant contribution, the Witten index, so that the elliptic genus is holomorphic and independent of $\bar{\tau}$. For simplicity let us assume that the central charge of the seed theory is a multiple of six, $c=6 k$. The elliptic genus is then a weak Jacobi form of weight 0 and index $k$. In particular, it transforms under $S L(2, \mathbb{Z})$ as

$$
\chi\left(\frac{a \tau+b}{c \tau+d}, \frac{z}{c \tau+d}\right)=e^{\frac{2 \pi i k c z^{2}}{c \tau+d}} \chi_{1}(\tau, z) .
$$

(See [19] for an introduction to weak Jacobi forms.) As before, we can write down the generating function for the elliptic genus of the symmetric orbifold,

$$
\mathcal{Z}=\prod_{n>0, m \geq 0, \ell} \frac{1}{\left(1-p^{n} q^{m} y^{\ell}\right)^{c(m n, \ell)}}=\exp \left(\sum_{L>0} \frac{p^{L}}{L} T_{L}^{\prime} \chi(q, y)\right)
$$

where in this case the Hecke operator is defined as

$$
T_{L}^{\prime} \chi(\tau, z)=\sum_{a d=N} \sum_{b=0}^{d-1} \chi\left(\frac{a \tau+b}{d}, a z\right)=\sum_{a d=N} d \sum_{m \geq 0, \ell} c(m d, \ell) q^{a m} y^{a \ell}
$$


We now want to specialize the elliptic genus to a partition function of the theory by specializing the value of $y$. Since $y$ is the variable that encodes information on the $U(1)$ charge of the states, we can use its specialization to spectrally flow the theory. In total there are 4 possible spin structures for the left movers on the torus, corresponding to RR versus NS sector with and without the insertion of $(-1)^{F}$ respectively. The spin structure which is anti-periodic in both space and time direction is simply a number, namely the Witten index of the theory. The other three spin structures are functions of $\tau$, and they are interchanged in the usual way by acting with $S L(2, \mathbb{Z})$.

From the elliptic genus we can then obtain the (left-moving) twisted partition function in the NS sector,

$$
Z^{\tilde{N} S, R}(\tau)=\operatorname{Tr}_{N S}(-1)^{F} q^{L_{0}-k / 4}
$$

by half a unit of spectral flow as

$$
Z_{N}^{\tilde{N} S, R}(\tau)=(-1)^{k N} q^{k N / 4} \chi_{N}(\tau, z=\tau / 2)
$$

Note that this is strictly speaking not the NS partition function, but its multiple by the number of right-moving RR ground states. Alternatively, we can also obtain the untwisted NS partition function

$$
Z_{N}^{N S, R}(\tau)=(-1)^{k N} q^{k N / 4} \chi_{N}(\tau, z=\tau / 2+1 / 2)
$$

As is expected of a $N S$ partition function, $Z_{N}^{N S, R}$ has simple transformation properties under the group $\Gamma_{\theta}=\left\langle S, T^{2}\right\rangle$ (see e.g. [20]). It is thus natural to expect that its phase diagram is determined no longer by the fundamental regions of $\Gamma$, but of $\Gamma_{\theta}$, such as

$$
\mathcal{F}_{\theta}: \quad \tau \in \mathbb{H}, \quad|\tau|>1, \quad|\operatorname{Re}(\tau)|<1 .
$$

Let us first get a rough picture of the phase diagram from general properties of NS partition functions. Since $Z^{N S, R}(\tau+1)=Z^{N S, R}(\tau)$, the phase diagrams of the two functions are simply shifted. The high temperature behavior of $Z_{N}^{N S, R}$ is obtained as in the bosonic case,

$$
Z_{N}^{N S, R}(\tau)=e^{2 \pi i k N / 4 \tau}+\ldots
$$

which shows that there is again a phase transition at $T=\frac{1}{2 \pi}$. This is not surprising, as this partition function simply counts the bosonic and fermionic states in the NS sector. The behavior of the twisted partition function is more interesting because of the insertion of $(-1)^{F}$, which can lead to cancellations between fermionic and bosonic states. In fact, since

$$
Z^{\tilde{N} S, R}(-1 / \tau)=Z^{R, R}(\tau)
$$

there is no divergence in the high temperature case, as

$$
Z^{\tilde{N} S, R}(0)=Z^{R, R}(i \infty)=N_{R R}
$$

the number of Ramond-Ramond ground states. For a symmetric orbifold theory with finite $N$ the $Z^{\tilde{N} S, R}$ thus does not diverge as we go to arbitrarily high temperatures. 
To get a rough idea of what will happen in the $N \rightarrow \infty$ case, let us find a lower bound for the number of these states by evaluating the elliptic genus for $z=0$. This gives

$$
\mathcal{Z}=\left(\prod_{n>0}\left(1-p^{n}\right)^{-\sum_{\ell} c(0, \ell)}\right)
$$

whose asymptotic behavior is

$$
Z_{N}^{R R} \sim e^{2 \pi \sqrt{\sum_{\ell} c(0, \ell) N / 24}} .
$$

Assuming that total number of Ramond-Ramond ground states has the same behavior as this lower bound, we see that asymptotic behavior for $N \rightarrow \infty$ is quite different. In particular this indicates that the rescaled free energy $f$ will not have a phase transition along the imaginary axis, which agrees with the phase diagram outlined above. The exact analysis below will confirm this.

Let us now analyze the free energy of the twisted NS partition function $\tilde{Z}^{\tilde{N} S, R}$ in the strict $N=\infty$ case in detail. As in the bosonic case, we introduce the shifted variable $\tilde{p}=p q^{-k / 2}$ and then factor out the lowest lying term $(1-p)^{n+1}$. In general however there will be more than single ground state, so that $n>0$. We would however still like to obtain the result by setting $p=1$ in the remainder of the expression. As a more careful analysis shows (see appendix A.4), the fact that there is more than one ground state adds a term $n \log N$ to the free energy. Even though this terms makes $\tilde{F}_{\infty}$ diverge, it is a subleading contribution to the rescaled free energy $f$. To obtain the phase diagram, we can thus neglect it and only consider

$$
\log \tilde{Z}_{\infty}^{\tilde{N} S, R}(\tau)=\sum_{a, n} \frac{1}{a} q^{a n k / 2} \sum_{m, \ell}^{\prime} c(m n, \ell) q^{a(m+\ell / 2)}=\sum_{a, n} \frac{1}{a} q^{a n k / 2} \sum_{b=0}^{n-1} \chi\left(\frac{a \tau+b}{n}, a \frac{\tau}{2}\right)
$$

for the twisted partition function.

We want to show that (2.37) converges in some region of the upper half plane. Since $Z^{\tilde{N} S, R}$ is invariant under the modular subgroup $\Gamma_{\theta}=\left\langle S, T^{2}\right\rangle$, we can assume that $\operatorname{Re}\left(\tau_{1}\right) \leq 1$. The problem is then essentially the same as in the bosonic case: we need to show that the Hecke transform of $\chi$ appearing in (2.37) does not grow too quickly, so that it can be suppressed by the prefactor $q^{a k n / 2}$.

Since we are interested in the behavior for $n$ large, it is natural again to use an $S$ transformation to obtain

$$
\chi\left(\frac{a \tau+b}{n}, a \frac{\tau}{2}\right)=\chi\left(-\frac{n}{a \tau+b}, \frac{n a \tau / 2}{a \tau+b}\right) e^{-2 \pi i k \frac{n(a \tau / 2)^{2}}{a \tau+b}} .
$$

In the bosonic case the leading contribution was then simply the vacuum. For the elliptic genus, we need to find which term $q^{m} y^{\ell}$ gives the dominant contribution. Not surprisingly, it turns out that we only need to worry about $a=1$, and the dominant term has $m=0$ and $\ell= \pm k$, the highest allowed value of $\ell$, and $b= \pm 1)-$ see appendix A.4. The total contribution in (2.37) is

$$
\exp \left[2 \pi \tau_{2} k n\left(-\frac{1}{4}+\frac{1}{4|\tau \pm 1|^{2}}\right)\right]
$$

so that (2.37) converges if and only if $|\tau+1|>1$ and $|\tau-1|>1$. The the free energy of the untwisted partition function $Z^{N S, R}$ thus converges in the fundamental region (2.31), and $f(\tau)$ in this region is given by $-\frac{k}{2}$. 


\subsection{Order parameters}

We would now like to analyze the contribution of the different twist sectors to the free energy. We expect that at low temperature, the main contribution will come from the untwisted sector which contains the vacuum. The higher the temperature, the more important the contributions of the twisted sectors will be. To make these statements more precise, we want to measure the contributions by constructing a family of order parameters. Not surprisingly, we will find that these order parameters undergo a phase transition at the Hagedorn temperature $f$ Their critical exponent turns out to be universal.

We want to define order parameters measuring the contribution of different twist sectors whose expectation values that can be easily evaluated. As was mentioned before, a twist sector in the theory is given by the conjugacy classes, so that

$$
(1)^{N_{1}}(2)^{N_{2}} \cdots(s)^{N_{s}}, \quad \sum_{n} n N_{n}=N \text {. }
$$

Before projection, a particular sector is therefore the tensor product of cycles of length $n$. It is therefore natural to define observables whose action factorizes over those cycles. We define a family of order parameters $P_{g}$ who act on a cycle of length $n$ by multiplication by $g(n)$, where $g$ is an arbitrary function of $n$. They then act on states of the twisted sector as

$$
P_{g}\left|\left\{N_{n}\right\}\right\rangle=\prod_{n} g(n)^{N_{n}}\left|\left\{N_{n}\right\}\right\rangle .
$$

The advantage of having an observable of the form (2.41) is that it is straightforward to obtain the generating function of its expectation values by repeating the original argument. Define $Z_{g}\left(S^{N} \mathcal{C}_{1} ; q, \bar{q}\right)$ as the trace of the symmetric orbifold theory with $P_{g}$ inserted. Since by construction $P_{g}$ factorizes over all twisted sectors, we can repeat the original derivation to obtain the generating function,

$$
\begin{aligned}
\mathcal{Z}_{g} & =\sum_{N \geq 0} p^{N} Z_{g}\left(S^{N} \mathcal{C}_{1} ; q, \bar{q}\right) \\
& =\sum_{N \geq 0} \sum_{\sum n N_{n}=N} \prod_{n>0} p^{n N_{n}} g(n)^{N_{n}} Z\left(S^{N_{n}} H_{(n)}^{Z_{n}} ; q, \bar{q}\right) \\
& =\prod_{n>0} \sum_{K \geq 0}\left(g(n) p^{n}\right)^{K} Z\left(S^{K} H_{(n)}^{Z_{n}} ; q, \bar{q}\right)
\end{aligned}
$$

where we have used the multiplication property of $P_{g}$ in the second line. We can then use the same reasoning as in the original argument to sum over $K$ to get

$$
\mathcal{Z}_{g}=\prod_{n>0} \prod_{m, \bar{m}}\left(1-g(n) p^{n} q^{m} \bar{q}^{\bar{m}}\right)^{-c_{n}(m, \bar{m})}
$$

By using the same trick as in section 2.2 we can then extract the expectation values of these order parameters for $N=\infty$,

$$
\left\langle P_{g}\right\rangle_{\infty}=\frac{\tilde{Z}_{\infty}^{g}}{\tilde{Z}_{\infty}} .
$$

\footnotetext{
†Strictly speaking, the canonical ensemble is no longer well-defined above this temperature. It is still possible however to work with finite $N$ and then take the limit $N \rightarrow \infty$ of the expectation values of the order parameters.
} 

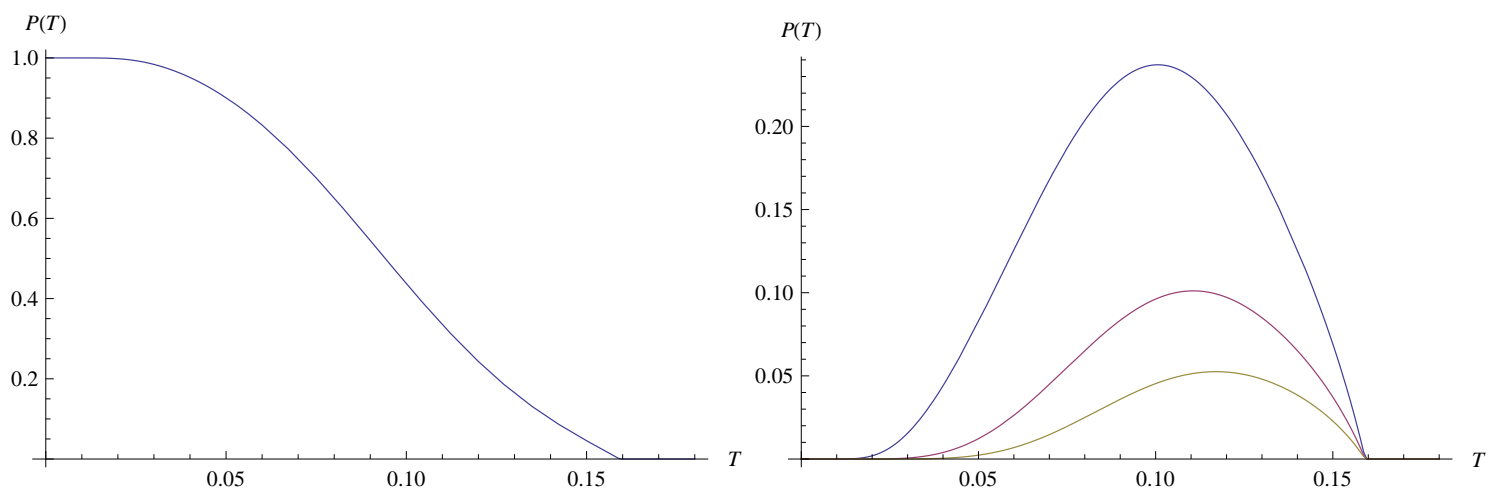

Figure 3: The free boson at self-dual radius: $\left\langle P_{\mathrm{utw}}\right\rangle_{\infty}$ as a function of $T$ (left), $\left\langle P_{(n)}\right\rangle_{\infty}$ for $n=2,3,4$ (right).

Let us now evaluate the expectation value for some of those order parameters. The contribution of the untwisted sector can be extracted by picking $g(1)=1, g(n>1)=0$ :

$$
\left\langle P_{\mathrm{utw}}\right\rangle_{\infty}=\tilde{Z}_{\infty}^{-1} \prod_{(m, \bar{m})>\left(-c_{L} / 24,-c_{R} / 24\right)}\left(1-q^{m+c_{L} / 24} \bar{q}^{\bar{m}+c_{R} / 24}\right)^{-d(m, \bar{m})} .
$$

We thus see that the contribution of the untwisted sector starts as 1 for $T=0$, decreases with growing temperature and vanishes once we cross the Hagedorn transition point. In particular this shows that $\left\langle P_{\mathrm{utw}}\right\rangle_{\infty}$ cannot be an analytic function of $\beta$. More precisely, the infinite product in (2.45) is regular, so that the behavior of $\left\langle P_{\mathrm{utw}}\right\rangle_{\infty}$ near the critical temperature is determined by the behavior of $\tilde{Z}_{\infty}^{-1}$. Using (2.19) we see that

$$
\left\langle P_{\mathrm{utw}}\right\rangle_{\infty}(T) \sim\left(T_{H}-T\right)^{1}
$$

i.e. the critical exponent of the order parameter is 1 . Note that although the vanishing of $Z^{-1}$ above the critical temperature is a generic feature of Hagedorn transitions, the exact value of the critical exponent depends on the theory. The value given in (2.46) is thus a statement on the subleading behavior of the growth of the number of states in symmetric orbifold theories.

To estimate the contribution of cycles of length $n$, we choose $g(1)=g(n)=1, g=0$ else. We include contributions of cycles of length 1 to ensure that for any finite $N \geq n$ there is a contribution. Subtracting the contribution of the untwisted sector we get

$$
\begin{aligned}
\left\langle P_{(n)}\right\rangle_{\infty}=\left\langle P_{(1),(n)}\right\rangle_{\infty}-\left\langle P_{(1)}\right\rangle_{\infty} & =\prod_{n^{\prime}>1, n^{\prime} \neq n, m, \bar{m}}\left(1-q^{m / n^{\prime}+n^{\prime} c_{L} / 24} \bar{q}^{\bar{m} / n^{\prime}+n^{\prime} c_{R} / 24}\right) d(m, \bar{m}) \delta_{m-\bar{m}}^{\left(n^{\prime}\right)} \\
& -\prod_{n^{\prime}>1, m, \bar{m}}\left(1-q^{m / n+n^{\prime} c_{L} / 24} \bar{q}^{\bar{m} / n^{\prime}+n^{\prime} c_{R} / 24}\right)^{d(m, \bar{m}) \delta_{m-\bar{m}}^{\left(n^{\prime}\right)}}
\end{aligned}
$$

We know that this vanishes for $T=0$ and $T=\frac{1}{2 \pi}$, so that there must be a maximum somewhere in between. Figure 3 shows $\left\langle P_{(n)}\right\rangle_{\infty}$ for a free boson at the self-dual radius. As expected, the higher twisted sectors achieve their maximal contribution at a higher temperature. 


\section{General families}

\subsection{Moving away from the orbifold point}

Our analysis so far was perfectly appropriate for problems in CFT. If we want to apply it to holographic duals of gravity theories, we need to modify it somewhat. In general, the holographic dual is not expected to be at the symmetric orbifold point, but rather at some other point in the moduli space of the theory where the orbifold has been resolved [21]. In the D1-D5 system for instance this means one has to deform the theory by exactly marginal operators in the twist 2 sector [22]. Even though there has been recent progress in performing computations in such deformed theories [23, 24, 25], to compute the spectrum of the deformed theory is still a very difficult task.

Let us thus turn the problem around. Instead of trying to deform the symmetric orbifold, we will analyze the phase diagram of general families of CFTs to see if they can possibly serve as a holographic dual to gravity theoriet The most restrictive point of view one can take is that the phase diagram of such a family $\left\{\mathcal{C}^{(N)}\right\}_{N \in \mathbb{N}}$ should look exactly like figure 2 , and that their free energy should be given by figure 1. For this to happen the free energy has to satisfy

$$
\lim _{N \rightarrow \infty} \frac{1}{N} \tilde{F}_{N}(\beta)=0,
$$

for all $\beta>2 \pi$. To rewrite this in terms of the states of the theory, use

$$
Z_{N}(\beta)=e^{-\beta \frac{\left(c_{L}+c_{R}\right) N}{24}}\left(1+\sum_{m, \bar{m}} \tilde{d}_{N}(m, \bar{m}) e^{-\beta(m+\bar{m})}\right),
$$

so that (3.1) becomes

$$
\lim _{N \rightarrow \infty} \frac{1}{N} \log \left(1+\sum_{m, \bar{m}} \tilde{d}_{N}(m, \bar{m}) e^{-\beta(m+\bar{m})}\right)=0 .
$$

Physically, the interpretation of (3.3) is clear. If the free energy is to look like figure 1 , then the low energy behavior must be given by the vacuum contribution alone. Although the contribution of the states above the vacuum is exponentially suppressed, there may be a lot of them, which is potentially enough to produce a Hagedorn behavior even at low temperature. This would then give a non-vanishing contribution to the free energy $f$. In particular it is possible that they can smooth out the Hawking-Page transition. Of course a gravity theory might also have a more complicated behavior than figures 11 and 2, Given such a behavior, one could again find criteria that holographic duals have to satisfy. For simplicity however let us restrict to (3.3).

Unfortunately (3.3) is not very transparent. In particular, some of the coefficients $\tilde{d}_{N}(m, \bar{m})$ we can choose freely, whereas others are fixed by modularity. To make (3.3) more transparent we return to the example of holomorphic theories.

\footnotetext{
${ }^{\ddagger}$ See [17] for a recent discussion of this question using different methods.
} 


\subsection{Holomorphic partition functions}

For holomorphic partition functions one can render (3.1) more transparent. Holomorphic partition functions appear in two contexts: for holomorphically factorisable theories and for chiral theories, where there are no right-moving fields and thus the holomorphic part by itself is the full partition function.

Let us thus consider a chiral theory of central charge $c=24 N$. Its partition function is a modular function with a pole of order $N$ at $q=0$. Such a function is uniquely determined by its polar and constant part, so that we need to fix only a finite number of coefficients $d(n)$. One way to see this is to use the Rademacher expansion of such a function.

For our purposes it will be more useful to follow [26]. Let

$$
J(q)=q^{-1}+196884 q+\ldots
$$

be the (modular invariant) partition function of the Monster CFT. It is related to the usual $j$-invariant by $J(\tau)=j(\tau)-744$. Acting with the Hecke operator $T_{n}^{\prime}$ we get

$$
T_{n}^{\prime} J(q)=q^{-n}+O(q) .
$$

It thus follows that we can write any holomorphic partition function as

$$
Z_{N}(\tau)=\sum_{n=0}^{N} \tilde{d}_{N}(n) T_{N-n}^{\prime} J(\tau)
$$

i.e. we can express all $\tilde{d}_{N}(n)$ in terms of the first $N$ coefficients.

One particular family of CFTs of interest are the proposed extremal CFTs of [26]. Their central charge is given by a multiple of 24 , and their partition function factorizes into a holomorphic and antiholomorphic part. They are called extremal because they contain the

minimal operator content compatible with modularity. In this case the $\tilde{d}_{N}(n)$ are given by the Virasoro descendants of the vacuum,

$$
\tilde{d}_{N}(n)=\left[\prod_{l=2}^{\infty} \frac{1}{1-q^{l}}\right]_{q^{n}}, \quad n=0, \ldots, N .
$$

The fact that we can write down modular invariant partition functions is of course not enough to guarantee the existence of extremal CFTs. It is in fact still an open question whether they exist for central charge bigger than 24 [27, 28, 29, 30]. We will simply use their partition functions as a template for our analysis. In fact it will turn out that one can greatly relax extremality condition on the spectrum and still get Hawking-Page transitions.

The proposed extremal CFTs are supposed to be dual to pure gravity on $A d S_{3}$, so that extremal partition function should exhibit Hawking-Page phase transitions. This was indeed shown in [9] by showing that for $N \rightarrow \infty$ there is a Lee-Yang type condensation of zeros of the partition function on the circle $|\tau|=1$. In view of (3.3) this is no surprise, as extremal partition functions contain the fewest states possible.

One can relax the extremality condition quite a lot and still get Hawking-Page transitions. More precisely, assume that for $n \leq N$ the coefficients grow as

$$
\tilde{d}(n)=e^{2 \pi \alpha n} e^{o(n)},
$$


with $o(n)$ some function such that $\lim _{n \rightarrow \infty} n^{-1} o(n)$. For simplicity we will also assume that $o(n)$ is monotonically growing. This function $o(n)$ may encode polynomial prefactors, but also Virasoro descendants of primaries, since (3.7) grows only like $\exp (\pi \sqrt{2 n / 3})$.

We can easily find a lower bound for the free energy by considering the contribution of states at level $N$, obtaining

$$
\log \tilde{F}(\beta) \geq(2 \pi \alpha-\beta) N+o(N) .
$$

It follows directly that for $\alpha>1(3.3)$ is violated so that there is no transition. On the other hand we will show that for $\alpha \leq 1$ there is a Hawking-Page transition.

To do this first note that for $n$ prime

$$
T_{n}^{\prime} J(i)=J(i n)+\sum_{b=0}^{n-1} J((i+b) / n) \leq J(i n)+\sum_{b=0}^{n-1} J(i / n)=(n+1) J(i n),
$$

where the inequality comes from the fact that the coefficients of $J(q)$ are real and positive. We can bound this expression by using

$$
J(i n) \leq e^{2 \pi n}+J(i)
$$

which again follows from the positivity of the coefficients. If $n$ is not prime, then we see from (2.5) that there are at most $n$ such sums with $d$ terms each. Since $d \leq n$ we can again bound their contribution by (3.11), getting in total

$$
T_{n}^{\prime} J(i) \leq n^{2}\left(e^{2 \pi n}+J(i)\right)
$$

For $\alpha \leq 1$ we can thus estimate

$$
e^{-2 \pi N} Z_{N}(i) \leq e^{-2 \pi N} \sum_{n=0}^{N} \tilde{d}(n)(N-n)^{2}\left(e^{2 \pi(N-n)}+J(i)\right) \leq N^{3} e^{o(N)}(J(i)+1),
$$

which satisfies (3.1). Note that this is compatible with the analysis of [9], where a condensation of zeros was found for the weaker bound $\alpha \leq 0.61$ and $o(n)=0$.

In principle one could also perform the same type of analysis for the elliptic genus, i.e. classify $N=2$ SCFT which exhibit Hawking-Page transitions. Note however that unlike the holomorphic case, not every combination of polar terms can be completed to a weak Jacobi form (see [20] in the context of pure supergravity). The coefficients of polar terms will thus have to satisfy certain constraints.

\section{Acknowledgements}

It is a pleasure to thank Costas Bachas and Jan Troost for initial collaboration on this project. I would also like to thank Miranda Cheng, Terry Gannon, Amit Giveon, Sean Hartnoll, Alex Maloney, Shiraz Minwalla, Shlomo Razamat and Andy Strominger for helpful discussions, and Alex Maloney and Matthias Gaberdiel for useful remarks on the draft. My research is supported by a John A. McCone Postdoctoral Fellowship. 


\section{A Appendix}

\section{A.1 A very brief remark on the Cardy formula}

In this appendix we will briefly rederive the Cardy formula [31], concentrating on its range of validity. The main purpose is to show that if the partition function has a large gap, then the Cardy formula is valid even for small values of $n$. For simplicity we restrict to the holomorphic case. \$ Let $Z(q)=\sum_{n} d(n) q^{n}$ be the modular invariant partition function. We can obtain $d(n)$ by contour integration,

$$
d(n)=\oint_{C} d q q^{-n-1} Z(q),
$$

where $C$ is an arbitrary closed contour that encloses the origin. We choose $q=e^{2 \pi i \tau}$, $\operatorname{Im} \tau=\sqrt{\frac{c}{24 n}}, \operatorname{Re} \tau \in[-\pi, \pi]$. We can then use modularity to write

$$
\int d \tau e^{-2 \pi i n \tau} Z(-1 / \tau)=\int d \tau e^{-2 \pi i n \tau} e^{\frac{c}{24} 2 \pi i / \tau}\left(1+O\left(e^{-i n_{0} L / \tau}\right)\right)
$$

The point is that for large $L$, the second term is negligible as long as $\tau$ remains of order 1. This is the case if $n \sim c$. We can then perform the usual saddle point approximation for the first term and obtain a maximum at $\tau_{0}=i \sqrt{\frac{c}{24 n}}$. Moreover the second derivative at $\tau_{0}$ is negative, real and proportional to $n^{3 / 2} c^{-1 / 2}$, so that for large $c$ the saddle point approximation is valid. In total we thus get

$$
d(n) \sim\left(\frac{c}{96 n^{3}}\right)^{1 / 4} e^{2 \pi \sqrt{c n / 6}}
$$

\section{A.2 Convergence of the free energy}

The free energy is given by $\tilde{F}_{\infty}$,

$$
\begin{array}{r}
\beta^{-1} \tilde{F}_{\infty}=\log \tilde{Z}_{\infty}=-\sum_{n>0} \sum_{m, \bar{m}}^{\prime} d(m, \bar{m}) \delta_{m-\bar{m}}^{(n)} \log \left(1-q^{m / n+n c_{L} / 24} \bar{q}^{\bar{m} / n+n c_{R} / 24}\right) \\
=\sum_{n>0} \sum_{m, \bar{m}}^{\prime} d(m, \bar{m}) \delta_{m-\bar{m}}^{(n)} \sum_{a=1}^{\infty} a^{-1} q^{a\left(m / n+n c_{L} / 24\right)} \bar{q}^{a\left(\bar{m} / n+n c_{R} / 24\right)} .
\end{array}
$$

We want to show that this expression converges if $\tau$ is in the fundamental domain $\mathcal{F}_{0}$, $|\tau|>1,|\operatorname{Re}(\tau)|<\frac{1}{2}$.

Some brief comments on convergence are in order. For fixed $n$, the sum over $m, \bar{m}, a$ converges absolutely, as can be seen by using $-\log (1-x) \leq \frac{x}{1-x}$ and the fact that the asymptotic behavior of $d(m, \bar{m})$ is determined by the Cardy formula. This allows us to exchange the summation over $m$ and $a$. Moreover, the divergent behavior of the sum is determined by large values of $n$ only, so that for the rest of the argument we can always assume that $n$ is large. For a start we will concentrate on the term $a=1$, as we will argue later on that this is the only important term.

\footnotetext{
${ }^{\S}$ See [32] for a discussion why right and left movers factorize for large $m$ and $\bar{m}$.
} 
Using

$$
\delta_{m-\bar{m}}^{(n)}=\frac{1}{n} \sum_{k=0}^{n-1} e^{2 \pi i(m-\bar{m}) k / n}
$$

we rewrite the $n$-th term of (A.4) as

$$
\frac{1}{n} \sum_{k=0}^{n-1} \sum_{m, \bar{m}} d(m, \bar{m}) e^{2 \pi i(m-\bar{m}) k / n} q^{\left(m / n+n c_{L} / 24\right)} \bar{q}^{\left(\bar{m} / n+n c_{R} / 24\right)}=\frac{1}{n} \sum_{k=0}^{n-1} Z_{1}\left(\frac{\tau+k}{n}\right) q^{n c_{L} / 24} q^{n c_{R} / 24} .
$$

We now claim that for $n$ large enough and $\tau \in \mathcal{F}_{0},\left|Z_{1}\left(\tau_{k}\right)\right| \leq\left|Z_{1}\left(\tau_{0}\right)\right|$ (where $\left.\tau_{k}=(\tau+k) / n\right)$. To show this, let us map each $\tau_{k}$ to its image $\hat{\tau}_{k}$ in $\mathcal{F}_{0}$. Clearly $\operatorname{Im}\left(\hat{\tau}_{0}\right)=\operatorname{Im}(\tau) \frac{n}{|\tau|^{2}}$. It is then straightforward to show that all the other $\hat{\tau}_{k}$ have smaller imaginary part . Since for $\operatorname{Im}(\tau)$ big enough $Z_{1}(\tau)$ is monotonically increasing, this shows the claim.

Using that $\operatorname{Im}\left(\hat{\tau}_{0}\right)$ is very large, we can thus bound (A.6) by

$$
\begin{aligned}
\exp \left(2 \pi \frac{c_{L} \operatorname{Im}\left(\hat{\tau}_{0}\right)+c_{R} \operatorname{Im}\left(\hat{\tau}_{0}\right)}{24}\right) \exp \left(-2 \pi \frac{n\left(c_{L} \operatorname{Im}(\tau)+c_{R} \operatorname{Im}(\tau)\right.}{24}\right) & \\
& =\exp \left(2 \pi \frac{n\left(c_{L}+c_{R}\right) \operatorname{Im}(\tau)}{24}\left(\frac{1}{|\tau|^{2}}-1\right)\right) .
\end{aligned}
$$

The coefficient of $n$ is negative if

$$
|\tau|^{2}>1
$$

in which case the sum converges. Including the terms with $a>1$ simply changes the temperature to $a \tau$, and the resulting sums in $n$ fall off exponentially in $a$, so that the sum over a converges.

\section{A.3 Degenerate ground states}

Let us briefly discuss the case of degenerate ground states. If there are $n+1$ ground states then the generating function is of the form

$$
F(p)=\sum_{N \geq 0} d_{N} p^{N}=\frac{1}{(1-p)^{n+1}} f(p)
$$

with $f(p)=\sum_{k \geq 0} a_{k} p^{k}$. Moreover define $f_{N}(p)=\sum_{k=0}^{N} a_{k} p^{k}$. We know that $f(p)$ and its first $n$ derivatives converge for $p=1$. This follows from the fact that the derivative of $\log f(p)$ converges, which can be shown by the same type of analysis used to show convergence of $f(1)$. We then have

$$
d_{N}=\frac{N^{n}}{n !}\left(f_{N}(1)+O\left(N^{-1}\right)\right) \quad N \rightarrow \infty .
$$

To see this, note that

$$
d_{N}=\sum_{k=0}^{N} a_{k}\left(\begin{array}{c}
N-k+n \\
n
\end{array}\right)=\left.\frac{1}{n !} \frac{d^{n}}{d p^{n}}\left(p^{N+n} f_{N}\left(p^{-1}\right)\right)\right|_{p=1}=\frac{N^{n}}{n !} f_{N}(1)+O\left(N^{n-1}\right)
$$

I To see this note that by choosing a suitable summation range for $k, \tau_{k}$ is always in the strip $\left|\operatorname{Re}\left(\tau_{k}\right)\right|<\frac{1}{2}$ with $\left|\operatorname{Re}\left(\tau_{k}\right)\right|>\left|\operatorname{Re}\left(\tau_{0}\right)\right|$. After the first $S$ transform its imaginary part is thus smaller than that of $\hat{\tau}_{0}$, and further modular transformations do not change this. 
From what we have said above $f_{N}$ at its derivatives at $p=1$ are bounded. It thus follows that the degeneracy of the ground states only contributes a polynomial prefactor. In particular, its contribution to the free energy is subleading and can be neglected after rescaling by $N$.

\section{A.4 The dominant contribution for the elliptic genus}

We need to find the term with the biggest contribution in the expansion of

$$
\sum_{b=0}^{n-1} \chi\left(-\frac{n}{a \tau+b}, \frac{n a \tau / 2}{a \tau+b}\right) e^{-2 \pi i k \frac{n(a \tau / 2)^{2}}{a \tau+b}} .
$$

Use

$$
\begin{aligned}
\operatorname{Im}\left(-\frac{n}{a \tau+b}\right) & =\frac{n a}{|a \tau+b|^{2}} \tau_{2} \\
\operatorname{Im}\left(\frac{n a \tau / 2}{a \tau+b}\right) & =\frac{b a n}{2|a \tau+b|^{2}} \tau_{2}, \\
\operatorname{Im}\left(\frac{n(a \tau / 2)^{2}}{a \tau+b}\right) & =\frac{n a \tau_{2}}{4}\left(1-\frac{b^{2}}{|a \tau+b|^{2}}\right) .
\end{aligned}
$$

The exponent of the absolute value of the term corresponding to $q^{m} y^{\ell}$ in (A.12) is

$$
-2 \pi \tau_{2} n a\left(\frac{m+b \ell / 2+\frac{k}{4} b^{2}}{|a \tau+b|^{2}}-\frac{1}{4}\right) .
$$

Let us now find the minimum of the fraction in the above expression. It is clear that $|\ell|$ must attain its maximal value $\sqrt{4 m k+k^{2}}$, so that

$$
\frac{4 m \pm 2 b \sqrt{4 m k+k^{2}}+k b^{2}}{4|a \tau+b|^{2}}=k \frac{(b \pm \sqrt{1+4 m / k})^{2}-1}{4|a \tau+b|^{2}}
$$

From the form of this expression we see that again we can concentrate on the case $a=1$. Remembering that $\left|\tau_{1}\right|<1$, it is then straightforward to see that the exponent is minimal for $m=0$ with $b= \pm 1$, and its contribution is

$$
\exp \left[2 \pi \tau_{2} k n\left(\frac{1}{4}+\frac{1}{4|\tau \pm 1|^{2}}\right)\right] .
$$

\section{References}

[1] J. M. Maldacena, The Large $N$ limit of superconformal field theories and supergravity, Adv. Theor. Math. Phys. 2 (1998) 231-252. [hep-th/9711200].

[2] O. Aharony, S. S. Gubser, J. M. Maldacena, H. Ooguri and Y. Oz, Large N field theories, string theory and gravity, Phys. Rept. 323 (2000) 183-386. [hep-th/9905111].

[3] C. Vafa, Instantons on D-branes, Nucl. Phys. B463 (1996) 435-442. hep-th/9512078. 
[4] E. Witten, On the conformal field theory of the Higgs branch, JHEP 9707 (1997) 003. hep-th/9707093.

[5] S. W. Hawking, D. N. Page, Thermodynamics of Black Holes in anti-De Sitter Space, Commun. Math. Phys. 87 (1983) 577.

[6] R. Dijkgraaf, J. M. Maldacena, G. W. Moore and E. P. Verlinde, A black hole farey tail, arXiv:hep-th/0005003.

[7] J. M. Maldacena and A. Strominger, AdS(3) black holes and a stringy exclusion principle, JHEP 9812 (1998) 005 arXiv:hep-th/9804085].

[8] J. Manschot and G. W. Moore, A Modern Farey Tail, arXiv:0712.0573 [hep-th].

[9] A. Maloney and E. Witten, Quantum Gravity Partition Functions in Three Dimensions, arXiv:0712.0155 [hep-th].

[10] R. Dijkgraaf, G. W. Moore, E. P. Verlinde and H. L. Verlinde, Elliptic genera of symmetric products and second quantized strings, Commun. Math. Phys. 185 (1997) 197 arXiv:hep-th/9608096].

[11] R. Dijkgraaf, Fields, strings, matrices and symmetric products, arXiv:hep-th/9912104.

[12] P. Bantay, Symmetric products, permutation orbifolds and discrete torsion, Lett. Math. Phys. 63 (2003) 209 arXiv:hep-th/0004025.

[13] J. de Boer, Large N Elliptic Genus and AdS/CFT Correspondence, JHEP 9905 (1999) 017 arXiv:hep-th/9812240.

[14] B. Sundborg, The Hagedorn Transition, Deconfinement and N=4 SYM Theory, Nucl. Phys. B 573 (2000) 349 [arXiv:hep-th/9908001].

[15] O. Aharony, J. Marsano, S. Minwalla, K. Papadodimas and M. Van Raamsdonk, The Hagedorn / deconfinement phase transition in weakly coupled large $N$ gauge theories, Adv. Theor. Math. Phys. 8 (2004) 603 arXiv:hep-th/0310285.

[16] S. F. Hassan and S. R. Wadia, Gauge theory description of D-brane black holes: Emergence of the effective SCFT and Hawking radiation, Nucl. Phys. B 526 (1998) 311 arXiv:hep-th/9712213.

[17] S. El-Showk, K. Papadodimas, Emergent Spacetime and Holographic CFTs, arXiv:1101.4163 [hep-th]].

[18] E. Witten, Anti-de Sitter space, thermal phase transition, and confinement in gauge theories, Adv. Theor. Math. Phys. 2 (1998) 505 arXiv:hep-th/9803131.

[19] M. Eichler and D. Zagier, The Theory of Jacobi Forms, Birkhäuser, 1985.

[20] M. R. Gaberdiel, S. Gukov, C. A. Keller, G. W. Moore, H. Ooguri, Extremal N=(2,2) $2 D$ Conformal Field Theories and Constraints of Modularity, CNTP Vol 2 No 4 (2008) 743 arXiv:0805.4216 [hep-th]]. 
[21] N. Seiberg, E. Witten, The D1 / D5 system and singular CFT, JHEP 9904 (1999) 017. hep-th/9903224].

[22] J. R. David, G. Mandal, S. R. Wadia, Microscopic formulation of black holes in string theory, Phys. Rept. 369 (2002) 549-686. [hep-th/0203048].

[23] A. Pakman, L. Rastelli and S. S. Razamat, Diagrams for Symmetric Product Orbifolds, JHEP 0910 (2009) 034 [arXiv:0905.3448 [hep-th]].

[24] S. G. Avery, B. D. Chowdhury and S. D. Mathur, Deforming the D1D5 CFT away from the orbifold point, JHEP 1006 (2010) 031 [arXiv:1002.3132 [hep-th]].

[25] S. G. Avery, B. D. Chowdhury and S. D. Mathur, Excitations in the deformed D1D5 CFT, JHEP 1006 (2010) 032 [arXiv:1003.2746 [hep-th]].

[26] E. Witten, Three-Dimensional Gravity Revisited, arXiv:0706.3359 [hep-th].

[27] M. R. Gaberdiel, Constraints on extremal self-dual CFTs, JHEP 0711 (2007) 087. arXiv:0707.4073 [hep-th]].

[28] M. R. Gaberdiel, C. A. Keller, Modular differential equations and null vectors, JHEP 0809 (2008) 079. arXiv:0804.0489 [hep-th]].

[29] D. Gaiotto, X. Yin, Genus two partition functions of extremal conformal field theories, JHEP 0708 (2007) 029. arXiv:0707.3437 [hep-th]].

[30] M. R. Gaberdiel, C. A. Keller, R. Volpato, Genus two partition functions of chiral conformal field theories, CNTP Vol 4 No 2 (2010) 295 [arXiv:1002.3371 [hep-th]].

[31] J. L. Cardy, Operator Content of Two-Dimensional Conformally Invariant Theories, Nucl. Phys. B270 (1986) 186-204.

[32] F. Loran, M. M. Sheikh-Jabbari, M. Vincon, Beyond Logarithmic Corrections to Cardy Formula, arXiv:1010.3561 [hep-th]. 